\title{
3D geometry measurement of hot cylindric specimen using structured light
}

Lorenz Quentin, Rüdiger Beermann, Andreas Pösch, Eduard Reithmeier, Markus Kästner 


\title{
3D Geometry Measurement of Hot Cylindric Specimen Using Structured Light
}

\author{
Lorenz Quentin ${ }^{\mathrm{a}}$, Rüdiger Beermann ${ }^{\mathrm{a}}$, Andreas Pösch $^{\mathrm{a}}$, Eduard Reithmeier ${ }^{\mathrm{a}}$, and Markus \\ Kästner ${ }^{\mathrm{a}}$ \\ ${ }^{a}$ Institute of Measurement and Automatic Control, Leibniz Universität Hannover, Nienburger \\ Strasse 17, Hannover, Germany
}

\begin{abstract}
We present a fringe projection system to measure glowing hot hybrid components in between production processes. For this a high power green light projector, based on TI DLP technology, is used to create the highest possible contrast between fringes on the red glowing specimen. It has a resolution of $1140 \mathrm{x} 912$ pixels with a maximum frame rate of 120 images per second for fast measurement. We use a green bandpass filter $(525 \mathrm{~nm})$ on the camera lens to block unwanted incoming radiation from the specimen caused by self-emission. Commercial measurement standards are not calibrated for temperatures other than $20^{\circ} \mathrm{C}$, so they cannot be used to validate measurement data at the required temperatures of up to $1000^{\circ} \mathrm{C}$ since thermal expansion invalidates the geometry specification from the calibration data sheet. In our first development we use a uniformly heated pipe made of stainless steel as a dummy specimen to examine the measured geometry data. A pyrometer measures the temperature of the pipe so the expansion can be easily calculated using the thermal expansion coefficient. Different impact and triangulation angles are investigated to identify the effects of hot ambient air on the measurement. The impact of the induced refractive index gradient is examined to check the need for pre-processing steps in the measurement routine.
\end{abstract}

Keywords: 3D measurement, optics, structured light, fringe projection, inline inspection, hot measurement object

\section{INTRODUCTION}

Process automation and objective quality control are vital in the production of high quality products. This helps to reduce the influence of human uncertainties. Within the Collaborative Research Centre (CRC) 1153 a process chain for hybrid components is developed. The components are required to be inspected in between each production step within the shortest possible time. Furthermore, to minimise energy consumption for re-heating, it is not desirable to wait for the component to cool down, e.g. after a hot smithing or rolling process. Optical $3 \mathrm{~d}$ geometry measurement techniques such as fringe projection suits this time requirement well. These systems are usually validated and calibrated by measurement standards. These again are tracable to national standards. Since the calibration devices typically need constant temperatures to maintain accuracy, they cannot be used to reference the geometry measurement of a hot component. The usage of a fringe projection systems (fps) as a stand alone measurement technique for hot components is discussed in this paper. We also discuss the effects of the inhomegenous refractive index field, induced by the temperature gradient in the ambient air. To get a better look at these effects, the system setup includes two cameras with different triangulation angles.

\section{EXPERIMENTAL SETUP}

A schematic and an image of the overall structure of the used fringe projection system are shown in figure 1 . It contains a single Wintech DLP Pro $4500^{1}$ projector with a resolution of 1140 x 912 pixels and two Prosilica $\mathrm{GT}^{2}$ CCD cameras with a resolution of $2336 \times 1752$ pixels and $2048 \times 2048$ pixels each. On both cameras we use LINOS Mevis-C lenses ${ }^{3}$ and optical bandpass filters (MidOpt, centre wavelength $525 \mathrm{~nm}, \mathrm{FWHM} 80 \mathrm{~nm}$ ). ${ }^{4}$ The

Further author information: (Send correspondence to L. Quentin)

E-mail: quentin@imr.uni-hannover.de, Telephone: +49511 7625816

Optical Measurement Systems for Industrial Inspection X, edited by Peter Lehmann, Wolfgang Osten, Armando Albertazzi Gonçalves Jr., Proc. of SPIE Vol. 10329, 103290U · @ 2017 SPIE CCC code: $0277-786 \mathrm{X} / 17 / \$ 18 \cdot$ doi: $10.1117 / 12.2269607$

Proc. of SPIE Vol. $10329103290 U-1$ 


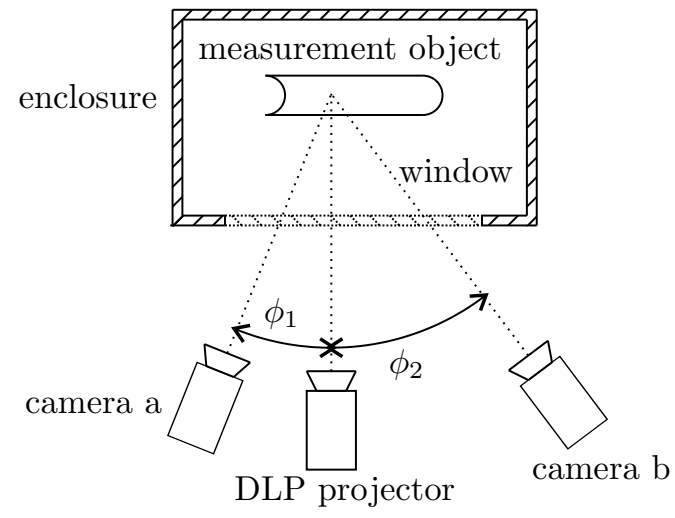

(a)

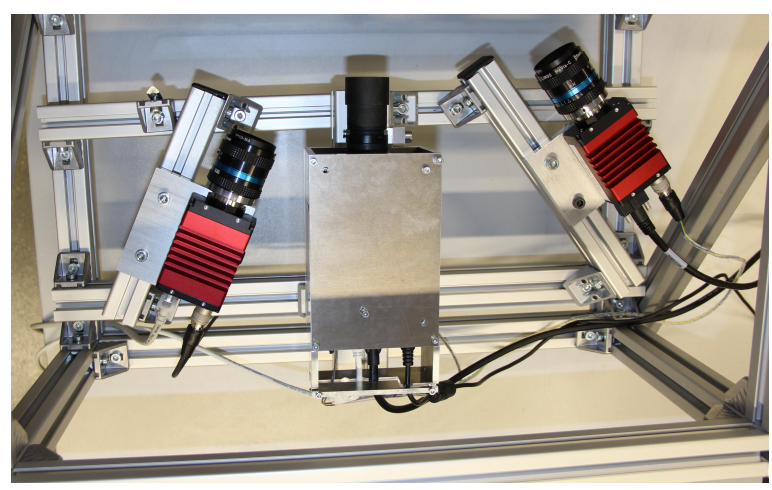

(b)

Figure 1: Setup of the measurement system. (a): schematic, (b): image.

projector is based on Texas Instruments DLP technology, it uses a high-power green LED at a maximum yield of $1330 \mathrm{~lm}$, with a frame rate of 120 images per second at full frame. Camera a is arranged at a triangulation angle between projector and camera of about $24^{\circ}$ (see $\phi_{1}$ in fig. 1a), whereas camera b is arranged at a slightly larger triangulation angle $\phi_{2}$ of about $38^{\circ}$. In further sections, the measurement system containing the DLP projector and camera a is referenced to by (fps a), while the system containing the projector and camera $\mathrm{b}$ is refered to as (fps b). A glass window made out of Schott BOROFLOAT is placed in between measurement system and the object to protect the camera lens from heat due to convection and prevent forced airflow. The object dummy is placed in a aluminium enclosure to generate a distraction free measurement enviroment. The fringe projection system is rigid bound to the glass window and the aluminium enclosure to reduce effects of imperfect calibration and others. The whole fringe projection - measurement object system can be rotated to measure through different parts of the density gradient field (see fig. 2).

\subsection{Calibration of the fringe projection system}

We use a fringe projection system based on Peng's multi-frequency phase-shift approach. ${ }^{5}$ Calibration uses methods based on Zhangs ${ }^{6}$ camera calibration and the findings of Pösch. ${ }^{7}$ Since coloured over-projection cannot be used with a single-colour projector, we use the phasemap of the uncalibrated projector to determine the correspondences between projector and cameras.

\subsection{Measurement plan}

We measure the hot dummy object in the positions given in figure 2 and denoted by table 1. A simple approximation of the density gradient is marked in the figure by the dotted line around the shaded measurement object.

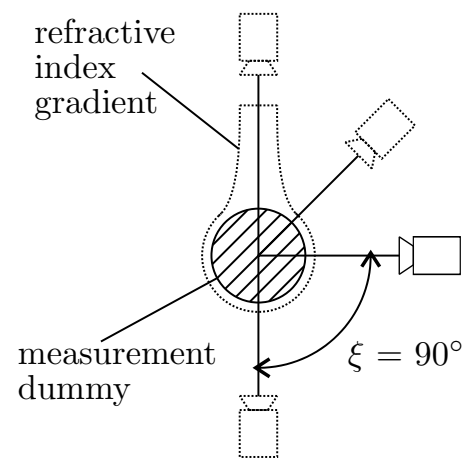

\section{positions of fringe projection system}

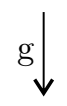

Figure 2: Illustration of different measurement poses to observe different parts of the refractive index gradient field.
Table 1: Angles of measurement poses.

\begin{tabular}{cc}
\hline $\begin{array}{c}\text { Number of } \\
\text { measurements }\end{array}$ & $\begin{array}{c}\text { Angle of } \\
\text { measurement } \xi\end{array}$ \\
\hline 3 & $0^{\circ}$ \\
3 & $90^{\circ}$ \\
3 & $135^{\circ}$ \\
3 & $180^{\circ}$ \\
\hline
\end{tabular}




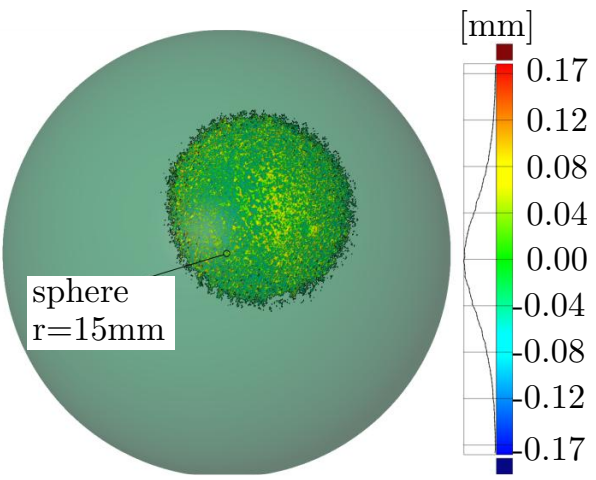

(a)

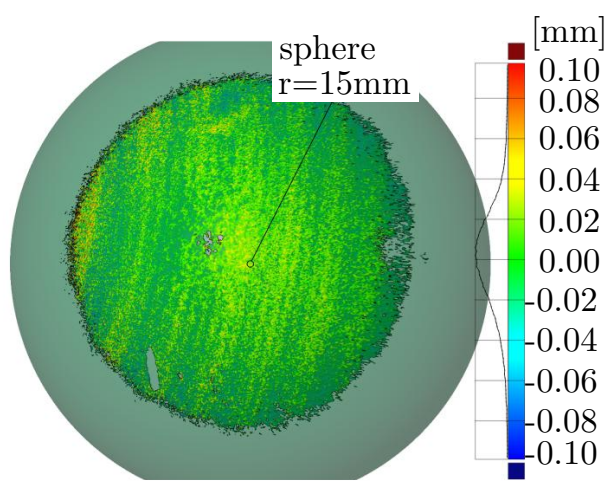

(b)

Figure 3: Deviations for the measured points of a spheric measurement standard and a fitted ideal sphere. (a): (fps a), (b): (fps b).

Table 2: Standard deviations and calculated radii of cold measurement object.

\begin{tabular}{ccc}
\hline & $\begin{array}{c}\text { Calculated radius } \\
/ \mathrm{mm}\end{array}$ & $\begin{array}{c}\text { Standard deviation } \\
/ \mathrm{\mu m}\end{array}$ \\
\hline (fps a) & 8.472 & 50 \\
(fps b) & 8.469 & 47 \\
\hline
\end{tabular}

It is dependend on the direction of the gravitational force, marked by g. The different width of the refractive index gradient caused by the density gradient will deflect the rays of light differently in each measurement position.

\section{RESULTS}

We use spheric measurement standards to validate the calibration of the fringe projection system for cold objects. The resulting points are then evaluated using GOM Inspect $2016 .{ }^{8}$ A sphere with a radius of $15 \mathrm{~mm}$ is fitted to the point cloud and the deviations are evaluated. The results for both fringe projection systems are displayed in figure 3. The legend is based on the $\pm 3 \sigma$ range of deviation to the ideal sphere, i.e. $99.7 \%$ of all measurement points lie within said distance. The standard deviation can easily be calculated from this to approximately $\pm 33 \mu \mathrm{m}$ for (fps a) and $\pm 60 \mu \mathrm{m}$ for (fps b). The calibration took place after the insertion of the glass window, so the image deformation effects of it can be assumed to be insignificant, as the calculated image undistortion compensates them.

\subsection{Measurement object}

We use a sandblasted pipe, made of stainless steel 1.4571 (X6CrNiMoTi17-12-2), as a measurement dummy (see fig. 4). Its diameter is about $17 \mathrm{~mm}$ and its wall thickness $2 \mathrm{~mm}$. On each end there are three screw-in tap holes to mechanically fix it to the heating tube. A plane spot is milled into the pipe to get significant radiation for a pyrometer temperature measurement. The average thermal expansion coefficient ${ }^{9}$ is $19.0 \times 10^{-6} \mathrm{~K}^{-1}$ for temperatures between $20^{\circ} \mathrm{C}$ and $500{ }^{\circ} \mathrm{C}$.

\subsection{Measurement of cold object}

To get a good base for further evaluation, the geometry of the dummy specimen is measured three times

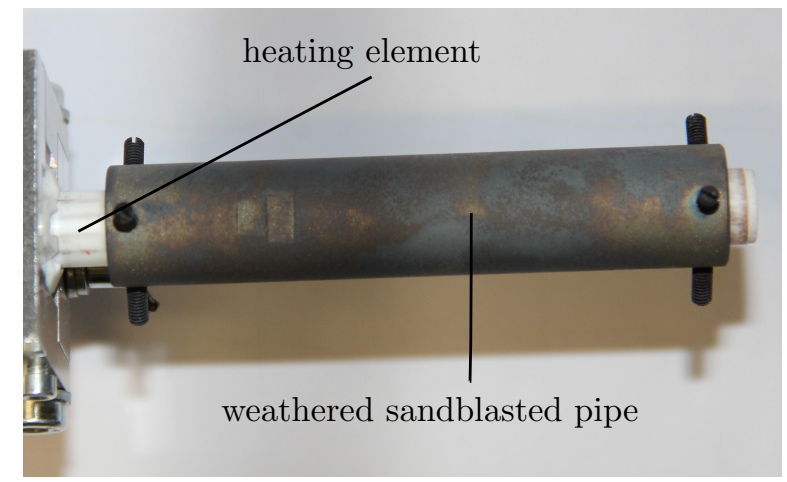

Figure 4: Image of measurement object. 


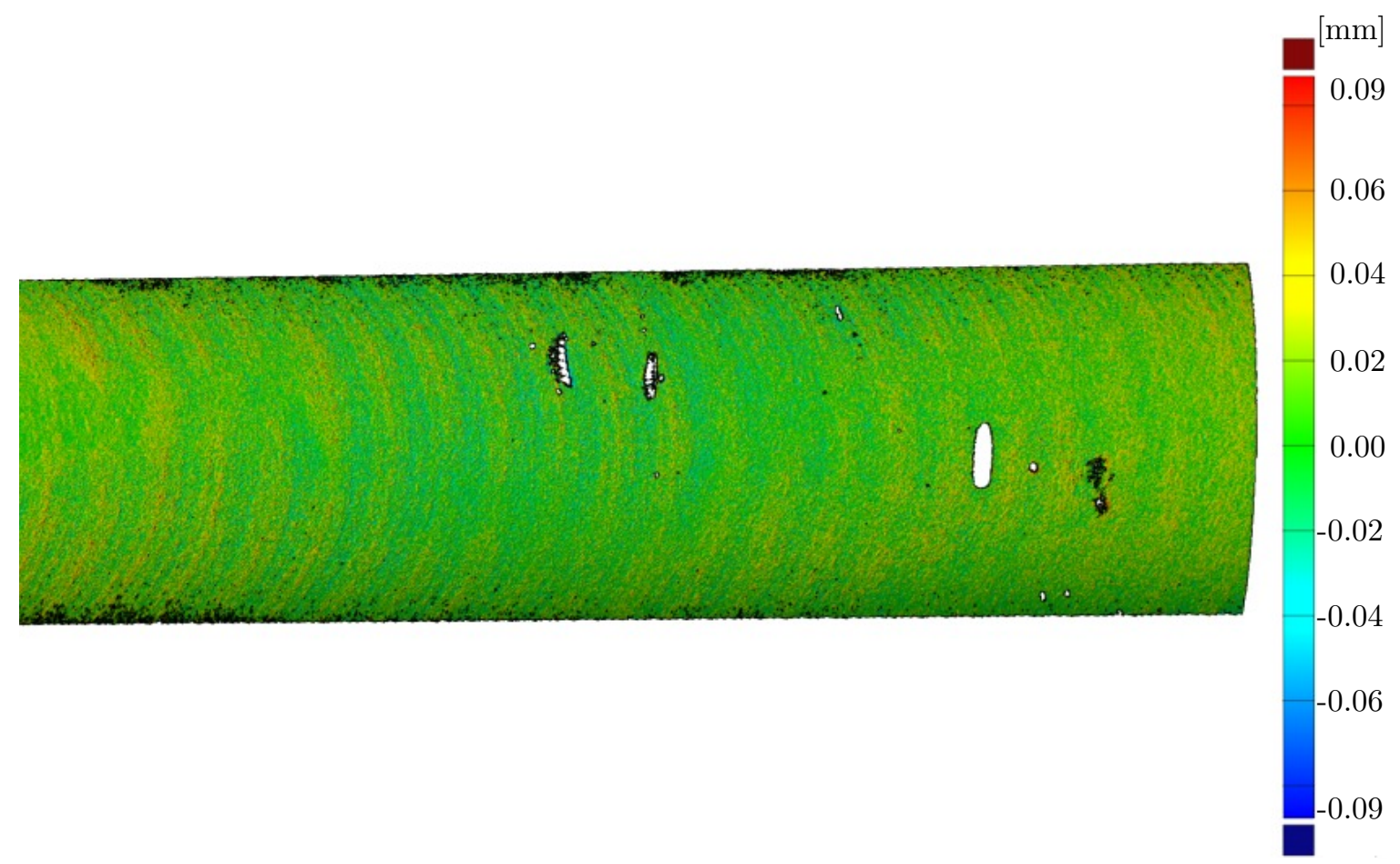

Figure 5: Deviation of measured geometry points of the cold measurement cylinder to fitted cylinder of (fps b).

in a cold state, i.e. at ambient temperature. The acquired points of all three measurements are averaged and a cylinder is fitted to the points. A deviation analysis is conducted, the result for (fps b) are displayed as an example in figure 5. Again the legend is based on the $\pm 3 \sigma$ range of deviation to the fitted cylinder and is within the referenced standard deviation of the fringe projection system. A couple of defects on the surface can be traced to particles on the camera lens or particles on the window. The radii and standard deviations of the fitted cylinders are displayed in table 2. The differences in radii are well within the calculated standard deviation respectivly the roughness of the sandblasted surface of the measurement dummy. To validate the correlation between both camera-projector-units, a cylinder is fitted into the measured geometry points on one fps with the radius acquired in the measurement of the other. A deviation analysis is conducted. The $3 \sigma$ range is about $\pm 140 \mu \mathrm{m}$, so well within the deviations of one fringe projection system itself. It can be assumed that both (fps a)re suited for the present measurement task.

\subsection{Measurement of hot object}

The dummy pipe is now heated from inside by a tube heater with about $90 \mathrm{~W}$ heating power and a maximum temperature of $1050{ }^{\circ} \mathrm{C}$. It heats the pipe to about $480^{\circ} \mathrm{C}$, measured by a pyrometer on the plane spot on the pipe. We assume the emissivity coefficient of weathered stainless steel ${ }^{10}$ of 0.85 to get the temperature at that particular spot. The change of the emissivity coefficient over temperature will be neglected, as well as the in-

Table 3: Radii and standard deviations of the hot measured object for (fps a).

\begin{tabular}{ccc}
\hline $\begin{array}{c}\text { Measurement } \\
\text { pose }\end{array}$ & $\begin{array}{c}\text { radius } \\
/ \mathrm{mm}\end{array}$ & $\begin{array}{c}\text { standard deviation } \\
/ \mathrm{\mu m}\end{array}$ \\
\hline $0^{\circ}$ & 8.576 & 50 \\
$90^{\circ}$ & 8.575 & 36 \\
$135^{\circ}$ & 8.560 & 40 \\
$180^{\circ}$ & 8.537 & 40 \\
\hline
\end{tabular}

Table 4: Radii and standard deviations of the hot measured object for (fps b).

\begin{tabular}{ccc}
\hline $\begin{array}{c}\text { Measurement } \\
\text { pose }\end{array}$ & $\begin{array}{c}\text { radius } \\
/ \mathrm{mm}\end{array}$ & $\begin{array}{c}\text { standard deviation } \\
/ \mu \mathrm{m}\end{array}$ \\
\hline $0^{\circ}$ & 8.566 & 43 \\
$90^{\circ}$ & 8.569 & 43 \\
$135^{\circ}$ & 8.569 & 47 \\
$180^{\circ}$ & 8.572 & 43 \\
\hline
\end{tabular}


homogeneous heating (and therefore inhomogenous expansion) due to different cooling effects. Deviation analysis of choses measurement points to the fitted cylinder are displayed in figures 6 and 7 as well as tables 3 and 4 .

\section{DISCUSSION OF RESULTS}

When comparing the standard deviations within one measurement setup across hot and cold specimen, the negeative influence of the higher temperature on the measurement uncertainty becomes obvious. To evaluate the radius properly, we first calculate the thermal expansion using the expansion equation for thin pipes

$$
\Delta r=\alpha r_{0} \Delta T
$$

with the radius of the cold pipe $r_{0}$, the thermal expansion coefficient $\alpha$ and the absolute temperature difference $\Delta T$. By using the stated equation, the average radius given in table 2 with $r_{o}=8.471 \mathrm{~mm}$ and $\Delta T=461 \mathrm{~K}$, we calculate a theoretical radius of the hot specimen $r_{T}$ of $8.513 \mathrm{~mm}$. Comparing the calculated radii given in tables 3 and 4 to the theoretical calculated radius from equation 1, a radius difference between $24 \mu \mathrm{m}$ and $63 \mu \mathrm{m}$ is observed. There is also a difference in calculated radii from different measurement poses and different triangulation angles. While the difference of calculated radii in different poses of (fps b) seems to be marginal ( $6 \mu \mathrm{m}$ or $14 \%$ of std), the difference in the measurements of (fps a) cannot be neglected ( $39 \mu \mathrm{m}$ or $94 \%$ of std) because the fitting cylinder function of GOM Inspect uses all marked points as a first guess and then enhances the fit by excluding points outside the $\pm 2 \sigma$-range. We assume the radius calculation to be more accurate than the specified standard deviation. The influence of the induced refractive index gradient is displayed in the measured

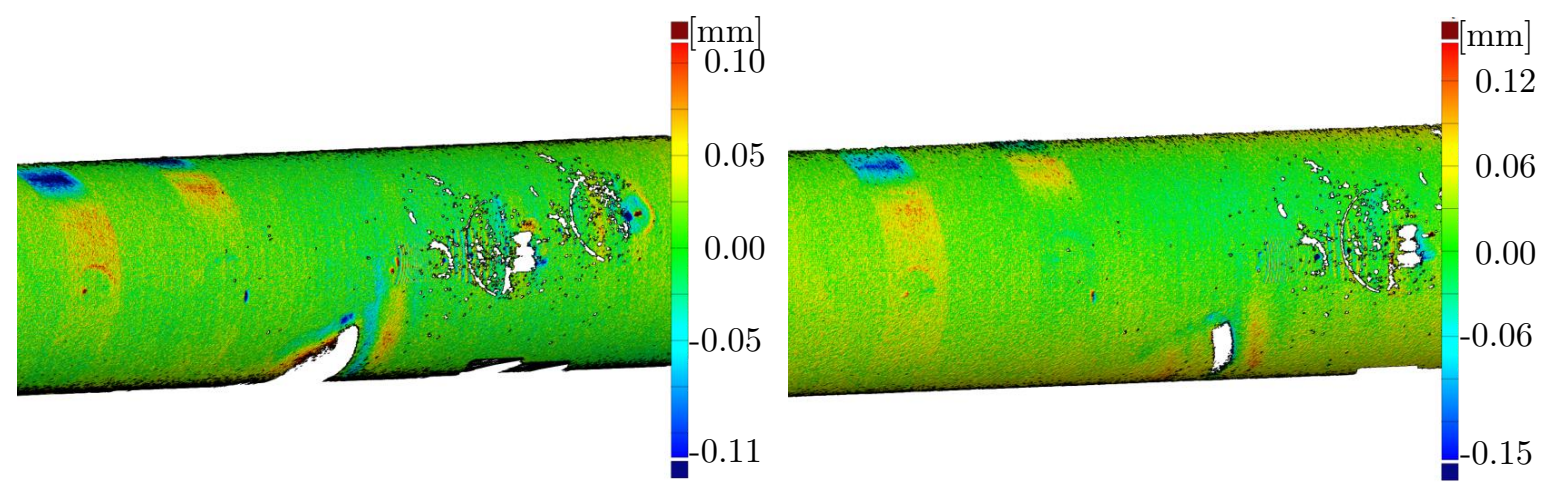

(a)

(b)

Figure 6: Deviation analysis of (fps a) to the fitted cylinder. (a): $\xi=90^{\circ}$, (b): $\xi=0^{\circ}$.

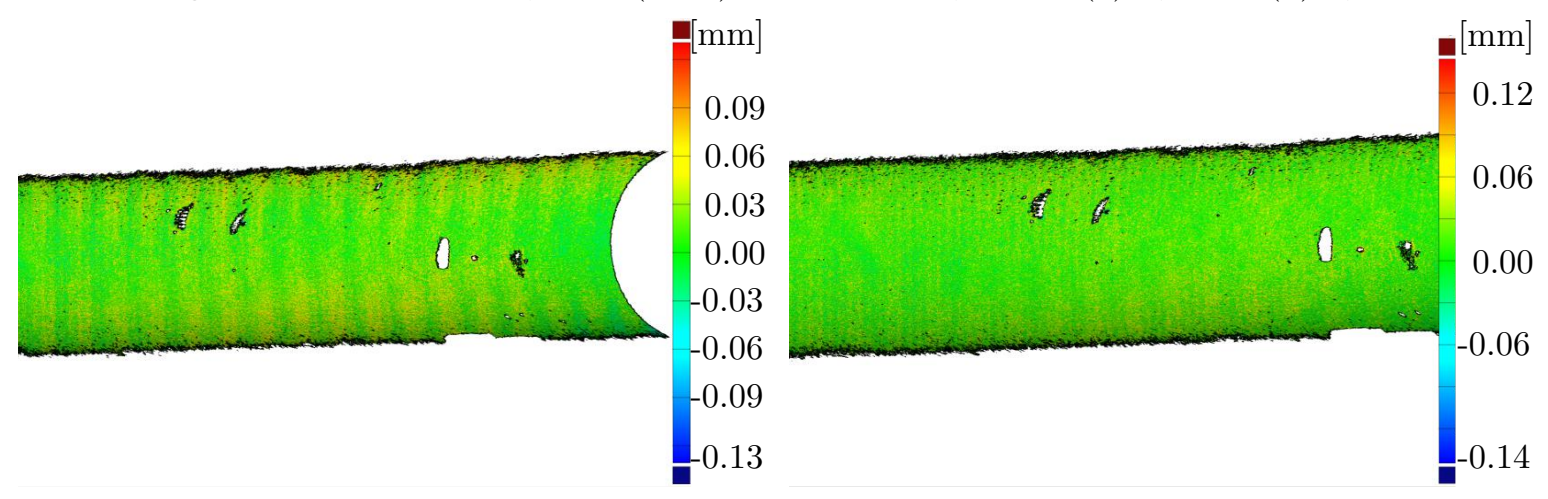

(a)

(b)

Figure 7: Deviation analysis of (fps b) to the fitted cylinder. (a): $\xi=180^{\circ}$, (b): $\xi=135^{\circ}$. 
radius at $\xi=180^{\circ}$ of (fps a) (see tab. 3). However, we observed some imperfections in the measured points of (fps a), marked by red and blue points in figure 6 , which could have a negative effect on the radius calculation. The negligence of volume expansion in the calculation of the reference radius of the hot measurement pipe could also reduce the accuracy of the made conclusion.

\section{CONCLUSION AND FUTURE WORK}

The presented fringe projection system is well suited to measure the geometry of hot specimen. The bandpass filters successfully block incoming radiation by the specimens self-emission. The results of the hot measurements are consistent within itself, but its validation is still interesting in regards to the missing reference. The comparision to a radius calculated by the thermal expansion equation for thin pipes while neglecting the volume expansion is a good initial guess but seems to be a stretch for accurate results.

In the future a measurement object heatable up to $1000^{\circ} \mathrm{C}$ would be a good start to better evaluate the effects of the gradient refractive index field caused by heated ambient air on optical $3 \mathrm{~d}$ measurements. The main objective should be to find a suitable reference system to validate the accuracy of hot $3 \mathrm{~d}$ geometry measurement systems.

\section{ACKNOWLEDGMENTS}

The results presented in this paper were obtained within the Collaborative Research Centre 1153 Process chain to produce hybrid high performance components by Tailored Forming in the subproject C5 Multiscale Geometry Inspection of Joining Zones. The authors would like to thank the German Research Foundation (DFG) for the financial and organisational support of this project. The authors would also like to thank Mr. Thomas Müller, Mr. Armin Dietz and Mr. Christopher Schindlbeck for their helpful input.

\section{REFERENCES}

[1] WintechDigital, "PRO4500 datasheet." http://www.wintechdigital.com/Upfiles/PR04500_Brochure. pdf. (Accessed: 06 April 2017).

[2] AlliedVision, "Prosilica GT." https://www.alliedvision.com/en/products/cameras.html?nc\# spectrum $\% 2 \mathrm{~F}-1 \% 2 \mathrm{FmaxFrameRate} \% 2 \mathrm{~F}-1 \% 2 \mathrm{FresolutionCalc} \% 2 \mathrm{~F}-1 \% 2 \mathrm{Fcol}$ or $\% 2 \mathrm{~F}-1 \% 2 \mathrm{FsensorSize} \% 2 \mathrm{~F}-1 \%$ $2 \mathrm{Fsensor} \% 2 \mathrm{~F}-1 \% 2 \mathrm{Fseries} \% 2 \mathrm{~F} 35 \% 2 \mathrm{Ftext} \% 2 \mathrm{~F} \% 2 \mathrm{Fintf} \% 2 \mathrm{~F}-1 \% 2 \mathrm{Finterfacefilter} \% 2 \mathrm{~F}-1 \% 2 \mathrm{~F}$. (Accessed: 06 April 2017).

[3] QIOPTIQ, "MeVis-C 1.6/25 datasheet." https://www.qioptiq-shop.com/out/Graphics/de/00120563_ 0.pdf. (Accessed: 06 April 2017).

[4] MidOpt, "BP525 Light Green Bandpass Filter." http://midopt.com/filters/bp525/. (Accessed: 06 April 2017).

[5] Peng, T., Gupta, S. K., and Lau, K., "Algorithms for constructing 3-d point clouds using multiple digital fringe projection patterns," Computer-Aided Design and Applications 2(6), 737-746 (2005).

[6] Zhang, Z., "A flexible new technique for camera calibration," Pattern Analysis and Machine Intelligence, IEEE Transactions on 22(11), 1330-1334 (2000).

[7] Poesch, A., Schlobohm, J., Matthias, S., and Reithmeier, E., "Rigid and flexible endoscopes for three dimensional measurement of inside machine parts using fringe projection," Optics and Lasers in Engineering 89, 178 - 183 (2017). 3DIM-DS 2015: Optical Image Processing in the context of 3D Imaging, Metrology, and Data Security.

[8] GOM, "GOM Inspect 2016." http://www.gom-inspect.com/de/index.php. (Accessed: 06 April 2017).

[9] ThyssenKrupp, "Werkstoffdatenblatt Edelstahl Rostfrei 1.4571." http://www.thyssenkrupp.at/files/ rsh/Werkstoffdatenblaetter/Stabstahl-Edelstahl-Rostfrei/1.4571.pdf. (Accessed: 06 April 2017).

[10] EngineeringToolbox, "Emissivity Coefficients." http://www.engineeringtoolbox.com/ emissivity-coefficients-d_447.html. (Accessed: 06 April 2017). 